\title{
Analysis of After-Hours Emergent Ophthalmic Surgery in the Ambulatory Surgery Center Setting
}

\section{Mark Obri*, Alexander Miller, Andrew Yazji, Emily Petrinec and David Miller}

Retinal Associates of Cleveland, Cleveland, Ohio, United States

*Corresponding Author: Mark Obri, Retinal Associates of Cleveland, Cleveland, Ohio, United States.
Received: March 30, 2021

Published: April 21, 2021

(C) All rights are reserved by Mark Obri., et al.

\section{Abstract}

Purpose: To evaluate the feasibility of emergent ophthalmologic surgeries in an ambulatory surgery center (ASC) care setting compared to routine ophthalmologic surgeries performed in an ASC.

Methods: A retrospective cohort study at a single ASC included an analysis of emergent surgical cases compared to non-emergent surgical cases. Data was provided by searching the ophthalmology practice's ASC case management database. An emergent case was defined as any case starting after 5:00 pm or any case completed during weekend on-call hours. Cases that were delayed and ran over the 5:00 pm time limit were excluded. The emergent cases were then analyzed based on the type of procedure performed and compared to the non-emergent ophthalmologic surgeries performed at the ASC.

Results: In 2018, 27 (34.2\%) of emergent cases were started after 5:00 pm and 52 (65.8\%) cases were performed during weekend call hours. This is compared to over 6113 ophthalmologic procedures done at the ASC in the same year during normal hours of operation. The division of total 2018 surgeries at this center was 3975 anterior segment eye surgeries, 1919 posterior segment surgeries, 211 oculoplastic surgeries, and 6 corneal surgeries. In total, 79 procedures were done emergently, representing $1.29 \%$ of all procedures. Posterior segment procedures were $100 \%$ of the emergent cases and constituted 64 retinal detachments (81\%), 9 endophthalmitis (11.4\%), 2 choroidal hemorrhages (2.5\%), 2 vitreous hemorrhages (2.5\%), and 2 retained lenses (2.5\%). No cases were refused, cancelled, or transferred elsewhere for emergent treatment, or because of lack of access to the facility by patients.

Conclusion: A total of 79 cases were successfully completed after-hours or during on-call weekend hours, representing $1.29 \%$ of the total amount of cases done in the 2018 calendar year. By allowing weekday 5:00 pm starts and weekend call hours for emergent cases, it appears the ASC setting is able to accommodate for all emergent ophthalmic cases, all of which were posterior segment surgeries, comprised mostly of retinal detachments.

Keywords: Ambulatory Surgical Centers (ASC); Ophthalmic Surgery

\section{Introduction}

Ambulatory Surgical Centers (ASC) are fast becoming one of the primary locations for surgery in the United States. In 2010, it was estimated that 25.7 million (53\%) ambulatory surgeries were performed in hospitals, and 22.5 million (47\%) were performed in ASCs, representing a large portion [1]. This change is due to a variety of factors. Advancing technology now allows physicians to perform surgeries without being in a hospital operating room. As of 2016, there are more than 6,100 ASCs in the U.S, with 5,519 that 
are Medicare-certified, allowing for increased access by a greater amount of people. Approximately $70 \%$ of ASCs are owned by independent physician groups, leading to preferential use due to ease of scheduling, usage, and access [2]. ASCs have the capabilities to host procedures once performed only in hospitals and have been found to have similar complications and readmission risks as the traditional hospital setting [3]. Benefits are also extended to patients in terms of the costs. According to Medicare, a patient pays $\$ 728$ USD for retinal detachment repair in a hospital setting and \$354 USD in an ASC. Many other procedures have the same trends [4]. In a 2020 study, emergent procedures performed at ASC's were shown to have decreased costs and time in the hospital compared to procedures performed in a hospital surgery setting. They found that noncomplicated, emergency surgeries in the outpatient setting could be performed with an equal to lower rate of readmission compared to a normal hospital setting [5]. Finally, ambulatory care settings have the ability to decrease total OR time and time under anesthesia for the patient, decreasing overall cost. Having fixed roles within an OR team at the ASC allows for a higher pace within the $\mathrm{OR}$, therefore increasing the volume of cases that can be managed [6].

There is a plethora of literature on the use of ASC's for elective surgeries, but there is a scarcity of studies done on the feasibility of using an ASC for emergent procedures, especially in the setting of ophthalmology. If it can be found that ASCs are feasible for emergent procedures, the continued benefit of cost savings and ease of access by patients and physicians could lead to more desirable patient outcomes. We analyzed one ASC to see whether it was feasible for an ASC to perform emergent ophthalmic procedures with similar complication outcomes and without need for patient transfer, compared to those performed during regular hours.

\section{Methods}

A retrospective cohort study was performed at a single ASC in Cleveland, Ohio. The study was performed via data analysis of emergent surgical cases compared to non-emergent surgical cases. The practice management database of the ASC was searched to provide all of the data from the year 2018. An emergent case was defined as any case starting after 5:00 pm during the week, or any case completed during weekend on-call hours. Any cases that were scheduled to end before 5:00 pm but were delayed (for any reason) and ran over the 5:00 pm time were excluded. The selected emergent cases were then analyzed based on the type of procedure that was performed and compared to the non-emergent ophthalmologic surgeries performed at the ASC.

\section{Results}

In 2018, there were 6113 ophthalmologic procedures performed at the ASC during normal operating hours. The division of total 2018 surgeries at this center was 3975 anterior segment eye surgeries, 1919 posterior segment surgeries, 211 oculoplastic surgeries, and 6 corneal surgeries (Figure 1). This is compared to 79 emergent procedures performed in 2018, representing 1.29\% percent of all procedures. 27 (34.2\%) of emergent cases were started after 5:00 pm and 52 (65.8\%) cases were performed during weekend call hours (Figure 1). Posterior segment procedures were $100 \%$ of the emergent cases, constituted of 64 retinal detachments, 9 endophthalmitis, 2 choroidal hemorrhages, 2 vitreous hemorrhages, and 2 retained lenses. The emergent cases were mostly retinal detachments (81\%). No cases were refused, cancelled or transferred elsewhere for further emergent treatment or due to patients' lack of access to the facility. Patient outcomes for procedures performed after 5:00 pm during the week or during weekend on-call hours were comparable to those performed during normal operating hours.

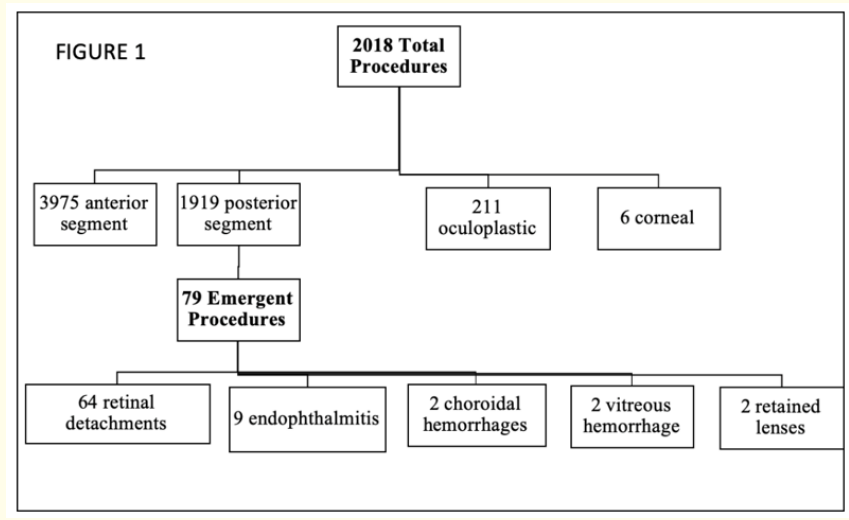

Figure 1

\section{Discussion and Conclusion}

By allowing start times after 5:00 pm during the week and weekend call hours for emergent cases, this study suggests the ASC setting is able to accommodate for emergent cases. This is 
an important observation, because if emergent procedures can be performed at an ASC, as was shown in this study, the benefits to physicians and patients are significant. Physicians are often required to deal with crowded operating rooms in hospitals, with limited scheduling and flexibility. This can be frustrating and unproductive. ASC's offer flexible scheduling because many are independently owned by physicians and are usually located away from crowded hospital complexes, allowing for greater ease of access. Patients also benefit a great amount. For example, the majority of emergent cases in this study were retinal detachments. According to Medicare reimbursements, a patient pays $\$ 728$ dollars for retinal detachment repair in a hospital setting and \$354 dollars in an ASC. Many other procedures have the same trends. This demonstrates the cost difference between an emergent surgery in an ASC versus a hospital. Overall, as the national discussion continues regarding patient healthcare costs and physician well-being, using an ASC for emergent procedures can potentially achieve both in the ophthalmologic setting, and possibly in other specialties. Ambulatory care centers allow for faster assessment and management of patients, allowing for a reduction in unnecessary inpatient hospital stays. Studies have also found that with proper training for surgeons, anesthesiologists, and OR support staff, emergent procedures can be done in an ASC with the same efficacy as in a hospital setting, but with higher patient satisfaction [7].

This review of ASC's as a potential setting for emergent ophthalmologic procedures has limitations. The most obvious shortcoming is that this is only a proof of concept for one ASC in the Cleveland area. The ophthalmology physician group at the studied ASC was willing to perform emergent procedures and had the necessary resources/infrastructure in place, which may not be the case for all ophthalmology groups or practices. This study also reviewed mostly retinal emergent cases, which limits the application to other ophthalmologic emergencies and to other medical specialties. Further limitations of ASC's include decreased ability to handle patients with severe comorbidities or procedures with extreme complications. In conclusion, this study provides valuable insight into the concept that an emergent ophthalmologic ASC has significant potential for benefits to both physicians and patients. However, further study is needed within the proposed topic.

\section{Key Points}

Can an Ambulatory Surgery Center handle emergent ophthalmic procedures as well as routine surgical settings?

\section{Findings}

The analysis found that this specific ASC had no problem managing emergent ophthalmologic procedures, with $1.29 \%$ of total cases emergent, and all with similar patient outcomes as compared to those performed in a routine surgical setting.

\section{Meaning}

This could lead to more usage of ASC's being used for emergent procedures, by providing a cheaper alternative to the hospital and increased patient and physician convenience.

\section{Summary Statement}

Ambulatory Surgical Centers are safe to use for emergent ophthalmologic cases, with increased ease of access, increased patient/physician satisfaction, and decreased cost, compared to routine surgical settings.

\section{Acknowledgement}

This material was presented in part at the 2019 meeting of the Association for Research in Vision and Ophthalmology.

\section{Financial Support}

None.

\section{Conflict of Interest}

No conflicting relationship exists for any author.

\section{Bibliography}

1. Hall MJ., et al. "Ambulatory surgery data from hospitals and ambulatory surgery centers: United States, 2010". National Health Statistics Reports 102 (2017): 1-15.

2. 2018 Ambulatory Surgery Center (ASC) Market: Projected to Increase from $\$ 36$ Billion in 2018, to $\$ 40$ Billion by (2020).

3. Tyler J Brolin., et al. "Neer Award 2016: Outpatient total shoulder arthroplasty in an ambulatory surgery center is a safe alternative to inpatient total shoulder arthroplasty in a hospital: a matched cohort study". Journal of Shoulder and Elbow Surgery 26.2 (2017): 204-208.

4. Medicare Procedure Price Lookup (2020).

5. Skues Mark A. "High-risk surgical procedures and semi-emergent surgical procedures for ambulatory surgery". Current Opinion in Anaesthesiology 33.6 (2020): 718-723. 
6. Fabricant PD., et al. "Cost Savings from Utilization of an Ambulatory Surgery Center for Orthopaedic Day Surgery". The Journal of the American Academy of Orthopaedic Surgeons 24.12 (2016): 865-871.

7. Franck L., et al. "[Feasibility study for ambulatory surgery in emergency]". Annales Francaises D'anesthesie et de Reanimation 32.6 (2013): 392-396.

\section{Assets from publication with us}

- Prompt Acknowledgement after receiving the article

- Thorough Double blinded peer review

- Rapid Publication

- Issue of Publication Certificate

- High visibility of your Published work

Website: www.actascientific.com/

Submit Article: www.actascientific.com/submission.php

Email us: editor@actascientific.com

Contact us: +919182824667 